\title{
Interactions between IncRNA TUG1 and miR-9 modulate the resistance of breast cancer cells to doxorubicin by regulating elF5A2
}

\section{Shuqian Wang}

Zhejiang University School of Medicine First Affiliated Hospital

\section{Mengjing Cheng}

Tongde Hospital Of Zhejiang Province

\section{Xiaoxiao Zheng}

Tongde Hospital Of Zhejiang Province

\section{Li Zheng}

Tongde Hospital Of Zhejiang Province

\section{Hao Liu}

Tongde Hospital Of Zhejiang Province

\section{Jianju Lu}

First Hospital of Jiaxing

Yu Liu

Zhejiang University School of Medicine First Affiliated Hospital

Wei Chen ( $\nabla$ wei_chen@zju.edu.cn )

Tongde Hospital Of Zhejiang Province https://orcid.org/0000-0002-0399-6216

\section{Research}

Keywords: TUG1, microRNA-9, breast cancer, doxorubicin resistance, elF5A2

Posted Date: December 17th, 2019

DOI: https://doi.org/10.21203/rs.2.19020/v1

License: (1) (1) This work is licensed under a Creative Commons Attribution 4.0 International License. Read Full License 


\section{Abstract}

Background: Breast cancer (BC) is one of the leading causes of cancer-related deaths. Chemoresistance of $B C$ remains a major unmet clinical obstacle. TUG1 (Taurine upregulated gene 1), a long noncoding RNA (IncRNA), and microRNAs (miRNA) are implicated in therapeutic resistance. However, the interactions between TUG1 and miRNAs that regulate doxorubicin (Dox) resistance in BC remain elusive.

Methods: Expression of TUG1 and miR-9 was measured by qRT-PCR (Quantitative real-time polymerase chain reaction). EIF5A2 (Eukaryotic translation initiation factor 5A-2) was detected by western blot. Transfection of siRNAs or miRNA inhibitors was applied to silence IncRNA TUG1, elF5A2 or miR-9. Cell viability, proliferation, and apoptosis were determined by CCK-8 (Cell counting kit-8), flow cytometry, and EdU (5-ethynyl-2'-deoxyuridine) assays, respectively. The regulatory relationship between TUG1 and miR-9 was determined by a luciferase assay.

Results: LncRNA TUG1 was highly expressed in BC tissues and positively associated with doxorubicin resistance in BC cell lines. SiRNA knockdown of TUG1 reversed doxorubicin resistance in MCF-7/ADR cells. Mechanistically, TUG1 acted as a 'sponge' for miR-9 and downregulated miR-9. Treatment with a miR-9 inhibitor blocked the effect of TUG1 siRNA, and knockdown of TUG1 inhibited the effects of miR-9. Furthermore, TUG1 inhibition of apoptosis induced by doxorubicin involved miR-9 targeting of elF5A2.

Conclusions: TUG1 modulates the susceptibility of BC cells to doxorubicin by regulating the expression of elF5A2 via a miR-9-dependent mechanism.

\section{Background}

Breast cancer (BC) is one of the leading causes of cancer-related deaths, has the highest incidence of malignant tumors among women, and is a significant public health concern[1]. Doxorubicin (Dox) is an anthracycline drug that is commonly used in the effective treatment of breast cancer, and resistance to Dox is a major challenge in breast cancer treatment[2,3]. Therefore, novel treatment approaches that enable clinicians and researchers to explore the potential mechanisms underlying BC chemoresistance and develop effective new strategies to enhance the efficacy of chemotherapy are urgently needed.

Long noncoding RNAs (LncRNAs) are an abundant and functionally diverse species of non-coding RNAs (ncRNAs) [4, 5]. LncRNAs play key roles in regulating gene expression related to drug resistance, growth, differentiation, and development [6, 7]. MicroRNAs (miRNAs) are small ncRNAs that negatively modulate gene expression[8, 9]. LncRNAs affect miRNA-mediated gene regulation by acting as competing endogenous RNAs, or natural miRNA sponges[10].

The IncRNA Taurine upregulated gene 1 (TUG1) directly binds to polycomb repressive complex 2 (PRC2) or PRC1 represses gene expression, and has been reported to participate in oncogenic processes[11, 12]. TUG1 acts as a key regulator of drug resistance by sponging miRNAs and affecting the expression of some cancer-related genes[13-15]. 
Here, using comparative profiling of IncRNAs between breast cancer tissues and adjacent normal tissues, we identified IncRNA TUG1 (LncTUG1) as an upregulated IncRNA in breast cancer. We also identified a negative correlation between TUG1 and miR-9. We had previously shown that miR-9 regulates daunorubicin resistance through regulation of eukaryotic translation initiation factor 5A-2 (EIF5A2)[16]. However, whether TUG1 can regulate the chemoresistance of breast cancer via similar mechanisms remains unknown.

We hypothesized that IncTUG1 directly binds to miR-9 and acts as an endogenous miR-9 sponge to inhibit miR-9 activity and contribute to Dox resistance. We further investigated the regulatory role of the IncTUG1/miR-9 interaction in doxorubicin resistance. Our results indicate that IncTUG1 may be a novel therapeutic target for breast cancer.

\section{Methods}

\section{Cell culture}

The BC cell lines MCF-7/ADR, MDA-MB-231, HCC1937, and MCF-7 were obtained from the Chinese Academy of Science Cell Bank (Shanghai, China) and cultured in RMPI 1640 medium (Gibco, Billings, MT, USA) supplemented with $10 \%$ fetal bovine serum and $1 \%$ penicillin-streptomycin solution (Gibco) at $37^{\circ} \mathrm{C}$ in a humidified atmosphere of $5 \% \mathrm{CO}_{2}$. Dox was purchased from Selleck Chemicals (Houston TX, USA) and dissolved in dimethyl sulfoxide.

\section{Small interfering (si)RNA transfection}

TUG1 siRNA, eIF5A2 siRNA, and negative-control siRNA were purchased from Ribobio (Guangzhou, China). MiR-9 inhibitors, miR-9 mimics, and negative control RNA oligos were obtained from Fulengen (Guangzhou, China) (miR-9 mimic: 5囚-UCUUUGGUUAUCUAGCUGUAUGA-3》 and 5》-

AUACAGCUAGAUAACCAAAGAUU-3®; miR-9 inhibitor: 5囚-UCAUACAGCUAGAUAACCAAAGA-3囚, negative control: 5区-CAGUACUUUUGUGUAGUACAA-3囚). Transfection was conducted using Lipofectamine 2000 (Thermo Scientific, Waltham, MA, USA), followed by gentle swirling, incubation and replacement of fresh culture medium.

\section{Cell Counting Kit-8 (CCK-8) assay}

$\mathrm{BC}$ cells were seeded in 96 -well plates at $1 \times 10^{\wedge} 4$ cells per well. Cells were treated with different concentrations of Dox for 48 h, CCK-8 reagent (Dojindo Laboratories, Tokyo, Japan) was added to each well at 1:100 dilution, and cells were incubated for $1 \mathrm{~h}$. Absorbance at $450 \mathrm{~nm}$ was measured using a microplate reader (MRX II; Dynex Technologies, Chantilly, VA, USA). The concentration at which each drug produced 50\% inhibition of growth (IC50) was estimated using a dose response curve. 


\section{Quantitative real-time polymerase chain reaction (qRT-PCR)}

Total RNA was extracted from BC cells using TRIzol $^{\text {TM }}$ Reagent (Thermo Scientific), and was reversetranscribed to CDNA using a TaqMan ${ }^{\text {TM }}$ miRNA kit or PrimeScript ${ }^{T M}$ RT kit. qRT-PCR assays were analyzed by the StepOnePlus ${ }^{\text {TM }}$ RT-PCR system (Thermo Scientific) using a TB SYBR ${ }^{\text {TM }}$ Premix Ex Taq ${ }^{\text {TM }}$ kit (TaKaRa Biotechnology, Dalian, China). The primers were as follows:

elF5A2: Forward: 5'-GCAGACGAAAUUGAUUUCATT-3';

Reverse: 5'-UGAAAUCAAUUUCGUCUGCTT-3';

TUG1: Forward: 5'-GCAGACGAAAUUGAUUUCATT-3';

Reverse: 5'-UGAAAUCAAUUUCGUCUGCTT-3';

miR-9: 5'-TCTTTGGTTATCTAGCTGTATGA-3'.

\section{EdU assay}

BC cells were seeded in 24-well plates at $1 \times 10^{5}$ cells per well. Cell proliferation was measured using a Click-iT® EdU Imaging kit (Invitrogen, Carlsbad, CA, USA), according to manufacturer instructions. Cell nuclei were stained blue, and EdU-positive cells were green.

\section{Apoptosis assay}

Apoptosis was determined using a fluorescein isothiocyanate (FITC)-conjugated annexin-V and a Propidium lodide (PI) kit, according to manufacturer instructions (BD Biosciences, San Jose, CA, USA). Cells were quantified by a CANTO ${ }^{\mathrm{TM}}$ II flow cytometer (BD Biosciences), and data were analyzed by FlowJo (Ashland, OR, USA).

\section{Western blotting}

Proteins were extracted from BC cell lines using RIPA lysis buffer (Beyotime, P0013B), isolated on sodium dodecyl sulfate polyacrylamide gels (12\%), and transferred onto polyvinylidene fluoride membranes. Membranes were incubated with antibodies recognizing elF5A2 or GAPDH (Abcam, Cambridge, UK) overnight at $4^{\circ} \mathrm{C}$. Goat anti-mouse/rabbit IgG secondary antibodies (Abcam, Cambridge, UK) were diluted at 1:2000. 


\section{Luciferase activity assays}

For the luciferase assay, $1.5 \times 10^{5}$ cells were seeded in a 24-well plate and were co-transfected with $100 \mathrm{ng}$ of either miR-9 mimics or negative control oligos, $30 \mathrm{ng}$ of firefly luciferase plasmid (containing either the wild-type or mutant TUG1 fragment), and 2 ng of pRL-TK vector (Promega, Madison, WI, USA), using Lipofectamine 3000 (Invitrogen) according to the manufacturer's recommendation. After $48 \mathrm{~h}$, the luciferase activity in the cells was measured using a luciferase assay kit (Promega) and normalized to the Renilla luciferase activity for each transfected well. Results represent independent experiments performed in triplicate.

\section{Statistical analyses}

Statistical analysis was performed using SPSS v18.0 (IBM, Armonk, NY, USA). All data are presented as the mean \pm SD and were assessed by two-tailed Student's t-tests. Any P-value of $P<0.05$ was considered to be statistically significant.

\section{Results}

\section{TUG1 is upregulated in breast cancer}

To investigate the potential involvement of IncRNAs in breast cancer, we examined the expression of cancer-related IncRNAs in 4 human breast cancer tissues and adjacent normal tissues using real-time PCR. Only TUG1 was highly expressed and significantly increased in breast cancer tissues (Fig. 1A). To validate this result, we explored the levels of TUG1 in additional breast cancer patients, and found that TUG1 expression in breast cancer tissue was significantly higher $(P<0.001, n=12)$ than in adjacent normal tissues (Fig. 1B). We evaluated Dox sensitivity in four breast cancer cell lines (MCF-7/ADR, MDAMB-231, HCC1937 and MCF-7) using the CCK-8 assay, and found that MCF-7 cells were the most sensitive to Dox, while MCF-7/ADR cells were the least sensitive (Fig. 1C). The expression of TUG1 was different in these four breast cancer cell lines, and TUG1 was most highly expressed in MCF-7/ADR cells, demonstrating an association of higher TUG1 expression with lower sensitivity to Dox (Fig. 1D-E).

\section{Knockdown of TUG1 inhibits Dox resistance in breast cancer}

We the explored the role of the IncRNA TUG1 as a mediator of breast cancer Dox resistance in vitro. Treatment of the four breast cancer cell lines with Dox (IC50) significantly increased the expression of the 
IncRNA TUG1(Fig. 2A). To investigate the function of TUG1 in regulating Dox sensitivity in breast cancer cells, we knocked down TUG1 using siRNA. The transfection efficiencies of three si-TUG1 oligos in these four breast cancer cell lines were detected using real-time PCR (Fig. 2B). We quantified cell viability in the presence of different concentrations of Dox in MCF-7/ADR and MCF-7 cells. Suppression of TUG1 inhibited Dox resistance in MCF-7/ADR and MCF-7 cells (Fig. 2C). The EdU assay performed on MCF7/ADR and MCF-7 cells confirmed these results (Fig. 2D-E). We then investigated whether the growth inhibition was caused by an increase in apoptosis. TUG1 siRNA treatment enhanced apoptosis in MCF7/ADR and MCF-7 cells, as determined by PI/Annexin V-FITC assay (Fig. 2F-G). These data demonstrate that IncTUG1 mediates Dox resistance, and may serve as a potential therapeutic target to overcome Dox resistance and enhance the benefits of Dox therapy in breast cancer.

\section{LncRNA TUG1 binds miR-9}

Having determined the role of IncRNA TUG1 in Dox resistance, we next explored mechanisms of TUG1 regulation by miRNAs. Using Starbase, we found the presence of a consensus-binding site for miR-9 in the TUG1 IncRNA. Co-transfection of a miR-9-overexpressing vector and a pmiR-GLO plasmid with the TUG1 WT sequence resulted in the downregulation of luciferase activity; this effect was reversed in HEK293T cells transfected with a mutated TUG1 (Fig. 3A). Consistent with this finding, miR-9 was increased after TUG1 was knocked down in MCF-7/ADR, MDA-MB-231, HCC1937, and MCF-7 breast cancer cell lines (Fig. 3B). In addition, knockdown of miR-9 in MCF-7 cells promoted Dox resistance, while overexpression of miR-9 in resistant MCF-7/ADR cells inhibited Dox resistance (Fig. 3C-D). Moreover, cell proliferation was accelerated after miR-9 was inhibited in MCF-7 cells. In contrast, the overexpression of miR-9 inhibited breast cancer proliferation (Fig. 3E). Furthermore, Inhibition or overexpression of miR-9 suppressed or promoted apoptosis in MCF-7 or MCF-7/ADR cells treated with Dox (Fig. 3F), respectively. Thus, overexpression of miR-9 can enhance Dox sensitivity in breast cancer cells.

\section{TUG1 inhibits Dox resistance by targeting miR-9 in vitro}

To determine whether the TUG1-mediated functional effects specifically depend on the expression of miR-9, we used a miR-9 inhibitor to examine whether the antitumor effect of TUG1 silencing could be blocked by miR-9 knockdown. Transfection of breast cancer cells with the miR-9 inhibitor significantly inhibited apoptosis induced by TUG1 siRNA (Fig. 4A-B). Moreover, miR-9 inhibitor significantly blocked the TUG1 knockdown-mediated enhancement of breast cancer cells proliferation (Fig. 4C-D). Additionally, the transduction of TUG1 siRNA resulted in enhanced susceptibility of breast cancer cells to Dox; this effect was reversed by pretreatment with the miR-9 inhibitor (Fig. 4E). TUG1 siRNA and miR-9 inhibitor notably decreased the expression of TUG1 and miR-9, respectively (Fig. 4F-G). 


\section{MiR-9 influences Dox resistance by targeting the TUG1 IncRNA in vitro}

In order to investigate whether the antitumor effects of miR-9 are affected by the sponge activity of the IncRNA TUG1, we examined Dox sensitivity in breast cancer cells with knockdown or overexpression of miR-9 after pre-treatment with TUG1 siRNA. The tumor-promoting effects of miR-9 silencing could be blocked by TUG1 siRNA, and the antitumor effect of miR-9 overexpression could be blocked by TUG1 knockdown (Fig. 5A). Consistently, TUG1 siRNA significantly blocked miR-9 silencing-mediated proliferation of MCF-7 and MCF-7/ADR cells (Fig. 5B-C). Furthermore, transduction of TUG1 siRNA rescued the suppression of apoptosis induced by miR-9 inhibitor in MCF-7 and MCF-7/ADR cells (Fig. 5DE). These findings demonstrate that miR-9 is involved in IncTUG1-mediated Dox resistance in breast cancer cells.

\section{EIF5A2 is the downstream target of the TUG1 IncRNA}

Our previous work demonstrated that elF5A2 is a direct target of miR-9. Therefore, we examined whether eIF5A2 lies downstream of the IncRNA TUG1 and is involved in regulating Dox resistance. We first examined whether IncRNA TUG1 can regulate the expression of elF5A2. Real-time PCR and western blot showed that the expression of elF5A2 in MCF-7 or MCF-7/ADR cells was downregulated significantly by TUG1 siRNA (Fig. 6A-B). CCK-8 assays revealed that elF5A2 knockdown resulted in much lower viability compared with control treatments, and the effects of TUG1 siRNA were disrupted when elF5A2 was knocked down (Fig. 6C). Treatment with an elF5A2 siRNA enhanced Dox-induced apoptosis in MCF7/ADR and MCF-7 cells, and this effect was not seen when TUG1 was knocked down (Fig. 6D-E). These data suggest that the effects of the IncRNA TUG1 in breast cancer cells are mediated by elF5A2, and that a IncTUG1/ miR-9/ elF5A2 axis regulates Dox sensitivity in breast cancer cells.

\section{Discussion}

In this study, we discovered the role of IncTUG1 in regulating Dox sensitivity in breast cancer cells, and we found that TUG1 regulates drug resistance of breast cancer cells through interacting with miR-9. These findings provide the first evidence that the Interaction between the IncRNA TUG1 and miR-9 mediates a pathway critical for the regulation of Dox resistance, and indicate that the IncRNA TUG1 may be a novel therapeutic target in breast cancer.

IncRNAs have important functions in many biological processes. In this study, we found that the IncRNA TUG1 exhibited high expression in clinical breast cancer tissues, suggesting that a correlation exists between IncTUG1 and the pathogenesis of breast cancer. Consistent with our findings, TUG1 has been shown to exert oncogenic effects in osteosarcoma, bladder cancer, esophageal cancer, and small cell lung cancer[15, 17, 18], whereas it is downregulated in non-small cell lung cancer[19]. TUG1 has been 
reported to promote the proliferation of ovarian cancer cells by down-regulating expression of Aurora kinase A (AURKA)[20]. In addition, a recent study demonstrated that expression of TUG1 is regulated by the Notch signaling pathway, and that TUG1 is highly expressed in GSCs and maintains stemness features of glioma cells[21].

Our findings support previous studies on the role of the TUG1 in the regulation of chemoresistance[13, 22]. TUG1 has been shown to promote Dox-resistance in osteosarcoma by suppressing Akt signaling [23]. However, whether TUG1 can regulate Dox sensitivity in breast cancer through miRNAs has not been previously investigated. To uncover the underlying molecular mechanisms by which TUG1 influences Dox sensitivity in breast cancer cells, we used bioinformatics methods to predict possible target genes. We found three main indications that show that miR-9 is a target gene of TUG1 in breast cancer; first, we found that miR-9 expression levels are significantly increased in TUG1 under-expressing breast cancer cell lines; second, a dual-luciferase assay showed that miR-9 reduced the luciferase activity of the wildtype TUG1 vector, but not that of a mutant TUG1 vector; third, the effects of TUG1 were reversed by the expression of miR-9, and miR-9 inhibitor treatment blocked the effects of TUG1 siRNA. Our study highlights the important regulatory relationships between miRNAs and IncRNAs in mediating Dox sensitivity in breast cancer.

Our previous work demonstrated that miR-9 rescues daunorubicin resistance by mediating elF5A2[16]. EIF5A2 is highly conserved throughout eukaryotic evolution and plays a role in mRNA translation, cellular proliferation, cellular differentiation, and inflammation[24-27]. Furthermore, elF5A2 is an important factor in the proliferation, metastasis and aggressiveness of cancer cells[28-30]. It has been suggested that elF5A2 acts as an oncogene and plays an important role in regulating drug resistance in breast cancer[31-33]. However, the role of the relationship between IncTUG1 and elF5A2 in breast cancer is not known. Here we report, for the first time, a new regulatory mechanism of elF5A2 expression. TUG1 knockdown increased Dox sensitivity and TUG1 mediated the regulatory role of elF5A2 in breast cancer cells. To our knowledge, this research is the first to demonstrate that elF5A2, as a downstream effector of TUG1, regulates Dox sensitivity in breast cancer, lending credence to our speculation that there is a IncTUG1-miR-9-EIF5A2 axis that can be manipulated to improve the efficacy of Dox in breast cancer. The mechanism by which elF5A2 regulates Dox resistance may involve induction of epithelial-mesenchymal transition, cytoskeletal rearrangement, angiogenesis, and metabolic reprogramming as reported[26, 3436].

Interestingly, we found that inhibition of TUG1 overcame the influences of miR-9-dependent susceptibility of BC cells to Dox. These observations revealed that TUG1 binds to miR-9, and that this interaction regulates the activity of both TUG1 and miR-9. TUG1 functions as a miR-9 sponge, and competes for binding of miR-9, directly interfering with the interaction of miR-9 and elF5A2, which blocked miR-9regulated Dox resistance in BC cells. Furthermore, miR-9 may degrade TUG1 after binding, but the regulation of TUG1 by miR-9 merits additional study.

\section{Conclusions}


Our study revealed a previously unappreciated regulatory mechanism by which IncTUG1 mediates Dox resistance in breast cancer, through targeting of the miR-9-EIF5A2 pathway. Furthermore, we emphasized the interaction between IncTUG1 and miR-9, which plays an important role in mediating Dox resistance in breast cancer. Specific blockage of IncTUG1 may be a potential therapeutic avenue to overcome Dox resistance in breast cancer treatment.

\section{Declarations}

\section{Acknowledgements}

We sincerely acknowledge members in the Chen laboratory for their technical assistance and helpful advices.

\section{Funding}

This study was supported by the Zhejiang Provincial Nature Science Foundation of China (LR20H160001); Key Project Co-constructed by Zhejiang Province and Ministry (WKJ-ZJ-1916); the National Natural Science Foundation of China (81972693, 81802383, 81972674 and 31900543); the Zhejiang Provincial Traditional Chinese Medicine Science and Technology Project (2020ZZ004); Zhejiang province Public Welfare Projects (No.2017C33089 and LGF18H200001).

\section{Availability of data and materials}

The datasets supporting the conclusions of this article are included within the article and its additional files.

\section{Authors' information}

\section{Affiliations}

Department of Breast Surgery, First Affiliated Hospital, School of Medicine, Zhejiang University, Hangzhou, 310003, China

Shuqian Wang \& Yu Liu

Department of Breast Surgery; The First Hospital of Jiaxing; Affiliated hospital of Jiaxing College; 1882\# Zhonghuan South Road; Jiaxing 314000, P. R. China

Jianju Lu 
Cancer Institute of Integrated Traditional Chinese and Western Medicine, Key Laboratory of Cancer Prevention and Therapy Combining Traditional Chinese and Western Medicine, Zhejiang Academy of Traditional Chinese Medicine, Tongde Hospital of Zhejiang province, Hangzhou, Zhejiang, 310012, China Mengjing Cheng, Xiaoxiao Zheng, Li Zhen, Hao Liu \& Wei Chen

\section{Contributions}

WSQ, CMJ, ZXX, ZL, and LH performed experiments and analyzed data. LJJ, LY and CW supervised the entire project. CMJ was a major contributor in writing the manuscript. All authors read and approved the final manuscript.

\section{Ethics approval and consent to participate}

This progect was approved by Ethics Committee of Tongde Hospital of Zhejiang province.

\section{Consent for publication}

Not applicable

\section{Competing interests}

The authors declare that they have no competing interests.

\section{Abbreviations}

AURKA

Aurora kinase $A$

EIF5A2

Eukaryotic translation initiation factor $5 \mathrm{~A}-2$

LncRNA

Long noncoding RNA

MiRNA

MicroRNA

NcRNAs

Non-coding RNAs

PRC2 
Polycomb repressive complex 2

TUG1

Taurine upregulated gene 1

\section{References}

1.

DeSantis CE, Ma J, Gaudet MM, Newman LA, Miller KD, Goding Sauer A, et al. Breast cancer statistics, 2019. CA Cancer J Clin. 2019.

2.

Young RC, Ozols RF, Myers CE. The anthracycline antineoplastic drugs. N Engl J Med. 1981;305:139-53. 3.

Arnason T, Harkness T. Development. Maintenance, and Reversal of Multiple Drug Resistance: At the Crossroads of TFPI1, ABC Transporters, and HIF1. Cancers (Basel). 2015;7:2063-82.

4.

lyer MK, Niknafs YS, Malik R, Singhal U, Sahu A, Hosono Y, et al. The landscape of long noncoding RNAs in the human transcriptome. Nat Genet. 2015;47:199-208.

5 .

Ulitsky I, Bartel DP. lincRNAs: genomics, evolution, and mechanisms. Cell. 2013;154:26-46.

6.

Bhan A, Soleimani M, Mandal SS. Long Noncoding RNA and Cancer: A New Paradigm. Cancer Res. 2017;77:3965-81.

7.

Malek E, Jagannathan S, Driscoll JJ. Correlation of long non-coding RNA expression with metastasis, drug resistance and clinical outcome in cancer. Oncotarget. 2014;5:8027-38.

8.

Filipowicz W, Bhattacharyya SN, Sonenberg N. Mechanisms of post-transcriptional regulation by microRNAs: are the answers in sight? Nat Rev Genet. 2008;9:102-14.

9.

Bartel DP. MicroRNAs: genomics, biogenesis, mechanism, and function. Cell. 2004;116:281-97. 10.

Tay Y, Rinn J, Pandolfi PP. The multilayered complexity of ceRNA crosstalk and competition. Nature. 2014;505:344-52.

11.

Khalil AM, Guttman M, Huarte M, Garber M, Raj A, Rivea Morales D, et al. Many human large intergenic noncoding RNAs associate with chromatin-modifying complexes and affect gene expression. Proc Natl Acad Sci U S A. 2009;106:11667-72.

12.

Yang L, Lin C, Liu W, Zhang J, Ohgi KA, Grinstein JD, et al. ncRNA- and Pc2 methylation-dependent gene relocation between nuclear structures mediates gene activation programs. Cell. 2011;147:773-88. 
13.

Yu G, Zhou H, Yao W, Meng L, Lang B. IncRNA TUG1 Promotes Cisplatin Resistance by Regulating CCND2 via Epigenetically Silencing miR-194-5p in Bladder Cancer. Mol Ther Nucleic Acids. 2019;16:257-71.

14.

Zhou Q, Hu T, Xu Y. Anticancer potential of TUG1 knockdown in cisplatin-resistant osteosarcoma through inhibition of MET/Akt signalling. J Drug Target. 2019:1-8.

15.

Niu Y, Ma F, Huang W, Fang S, Li M, Wei T, et al. Long non-coding RNA TUG1 is involved in cell growth and chemoresistance of small cell lung cancer by regulating LIMK2b via EZH2. Mol Cancer. 2017;16:5. 16.

Liu Y, Lei P, Qiao H, Sun K, Lu X, Bao F, et al. miR-9 Enhances the Chemosensitivity of AML Cells to Daunorubicin by Targeting the EIF5A2/MCL-1 Axis. Int J Biol Sci. 2019;15:579-86.

17.

Ma B, Li M, Zhang L, Huang M, Lei JB, Fu GH, et al. Upregulation of long non-coding RNA TUG1 correlates with poor prognosis and disease status in osteosarcoma. Tumour Biol. 2016;37:4445-55.

18.

Han Y, Liu Y, Gui Y, Cai Z. Long intergenic non-coding RNA TUG1 is overexpressed in urothelial carcinoma of the bladder. J Surg Oncol. 2013;107:555-9.

19.

Zhang EB, Yin DD, Sun M, Kong R, Liu XH, You LH, et al. P53-regulated long non-coding RNA TUG1 affects cell proliferation in human non-small cell lung cancer, partly through epigenetically regulating HOXB7 expression. Cell Death Dis. 2014;5:e1243.

20.

Li T, Chen Y, Zhang J, Liu S. LncRNA TUG1 promotes cells proliferation and inhibits cells apoptosis through regulating AURKA in epithelial ovarian cancer cells. Medicine. 2018;97:e12131.

21.

Katsushima K, Natsume A, Ohka F, Shinjo K, Hatanaka A, Ichimura N, et al. Targeting the Notch-regulated non-coding RNA TUG1 for glioma treatment. Nat Commun. 2016;7:13616.

22.

Li Q, Song W, Wang J. TUG1 confers Adriamycin resistance in acute myeloid leukemia by epigenetically suppressing miR-34a expression via EZH2. Biomed Pharmacother. 2019;109:1793-801.

23.

Hu T, Fei Z, Su H, Xie R, Chen L. Polydatin inhibits proliferation and promotes apoptosis of doxorubicinresistant osteosarcoma through LncRNA TUG1 mediated suppression of Akt signaling. Toxicol Appl Pharmacol. 2019;371:55-62.

24.

Mathews MB, Hershey JW. The translation factor elF5A and human cancer. Biochim Biophys Acta. 2015;1849:836-44.

25. 
Zender L, Xue W, Zuber J, Semighini CP, Krasnitz A, Ma B, et al. An oncogenomics-based in vivo RNAi screen identifies tumor suppressors in liver cancer. Cell. 2008;135:852-64.

26.

Cao TT, Lin SH, Fu L, Tang Z, Che CM, Zhang LY, et al. Eukaryotic translation initiation factor 5A2 promotes metabolic reprogramming in hepatocellular carcinoma cells. Carcinogenesis. 2017;38:94-104. 27.

Ganapathi M, Padgett LR, Yamada K, Devinsky O, Willaert R, Person R, et al. Recessive Rare Variants in Deoxyhypusine Synthase, an Enzyme Involved in the Synthesis of Hypusine, Are Associated with a Neurodevelopmental Disorder. Am J Hum Genet. 2019;104:287-98.

28.

Chen Z, Yu T, Zhou B, Wei J, Fang Y, Lu J, et al. Mg(II)-Catechin nanoparticles delivering siRNA targeting EIF5A2 inhibit bladder cancer cell growth in vitro and in vivo. Biomaterials. 2016;81:125-34. 29.

Zhu W, Cai MY, Tong ZT, Dong SS, Mai SJ, Liao YJ, et al. Overexpression of EIF5A2 promotes colorectal carcinoma cell aggressiveness by upregulating MTA1 through C-myc to induce epithelialmesenchymaltransition. Gut. 2012;61:562-75.

30 .

Li Y, Fu L, Li JB, Qin Y, Zeng TT, Zhou J, et al. Increased expression of EIF5A2, via hypoxia or gene amplification, contributes to metastasis and angiogenesis of esophageal squamous cell carcinoma. Gastroenterology. 2014;146:1701-13. e1709.

31.

Liu Y, Du F, Chen W, Yao M, Lv K, Fu P. EIF5A2 is a novel chemoresistance gene in breast cancer. Breast Cancer. 2015;22:602-7.

32.

Liu J, Wang P, Zhang P, Zhang X, Du H, Liu Q, et al. An integrative bioinformatics analysis identified miR375 as a candidate key regulator of malignant breast cancer. J Appl Genet. 2019;60:335-46.

33.

Post AEM, Bussink J, Sweep F, Span PN. Changes in DNA damage repair gene expression and cell cycle gene expression do not explain radioresistance in tamoxifen-resistant breast cancer. Oncol Res. 2019. 34.

Liu Y, Liu R, Fu P, Du F, Hong Y, Yao M, et al. N1-Guanyl-1,7-Diaminoheptane Sensitizes Estrogen Receptor Negative Breast Cancer Cells to Doxorubicin by Preventing Epithelial-Mesenchymal Transition through Inhibition of Eukaryotic Translation Initiation Factor 5A2 Activation. Cell Physiol Biochem. 2015;36:2494503.

35.

Tang DJ, Dong SS, Ma NF, Xie D, Chen L, Fu L, et al. Overexpression of eukaryotic initiation factor 5A2 enhances cell motility and promotes tumor metastasis in hepatocellular carcinoma. Hepatology. 2010;51:1255-63.

36. 
Li S, Ma Y, Xie C, Wu Z, Kang Z, Fang Z, et al. EphA6 promotes angiogenesis and prostate cancer metastasis and is associated with human prostate cancer progression. Oncotarget. 2015;6:22587-97.

\section{Figures}

\section{Figure 2}

TUG1 is upregulated in breast cancer. (A) Real-time PCR analysis was used to determine the expression levels of IncRNAs in 4 human breast cancer tissues and adjacent normal tissues. (B) Validation of the expression of IncRNA TUG1 expression. The IncRNA TUG1 expression levels in breast cancer tissues and adjacent normal tissues $(n=15)$, as measured by real-time PCR. (C) Viability of breast cancer cell lines under different concentrations of Dox, according to the CCK-8 assay. The IC50 of MCF-7/ADR, MDA-MB231, HCC1937, and MCF-7 were $33.6 \mu \mathrm{g} / \mathrm{ml}, 2.6 \mu \mathrm{g} / \mathrm{ml}, 1.4 \mu \mathrm{g} / \mathrm{ml}, 0.9 \mu \mathrm{g} / \mathrm{ml}$, respectively. (D) LncRNA TUG1 expression in breast cancer cell lines, as examined by qPCR. $P=0.0011 \mathrm{vs}$. the adjacent group using the Student's t test. (E) The correlation between the relative expression of IncRNA TUG1 and the IC50 of Dox.

\section{Figure 3}

Knockdown of TUG1 inhibits Dox resistance in breast cancer. (A) Relative expression of IncRNA TUG1 in breast cancer cell lines after Dox treatment. (B) Transfection efficacies of four si-TUG1 oligos in breast cancer cell lines. (C) The viability of MCF-7 and MCF-7/ADR cells transfected with si-TUG1 or negative control under different concentrations of Dox. (D) Proliferation of MCF-7 and MCF-7/ADR cells transfected with si-TUG1 or negative control and treated with Dox, according to the EdU assay. (E) The number of EdU-positive cells was counted. (F) Apoptosis of MCF-7 and MCF-7/ADR cells transfected with si-TUG1 or negative control and treated with Dox, according to the PI/Annexin V-FITC assay. (G) The quantification of apoptosis ratio of MCF-7 and MCF-7/ADR cells. ${ }^{*} P<0.05$, Dox group vs. negative control group, \#P<0.05, \#\#P<0.001, TUG1 siRNA+ Dox group vs. Dox group.

\section{Figure 5}

LncRNA TUG1 binds miR-9. (A) Starbase predicted binding between IncRNA TUG1 and miR-9. Luciferase activity decreased in the IncRNA TUG1 WT group. (B) LncRNA TUG1 knockdown upregulated miR-9 expression in breast cancer cell lines. (C) Transfection efficacies of miR-9 inhibitor or miR-9 mimics in MCF-7 and MCF-7/ADR cells. (D) Viability of MCF-7 or MCF-7/ADR cells transfected with miR-9 inhibitor or miR-9 mimics, under different concentrations of Dox. (E) Proliferation of MCF-7 or MCF-7/ADR cells transfected with miR-9 inhibitor or miR-9 mimics and treated with Dox according to the EdU assay. The 
number of EdU-positive cells was counted. (F) Apoptosis of MCF-7 and MCF-7/ADR cells transfected with miR-9 inhibitor or miR-9 mimics and treated with Dox according to the PI/Annexin V-FITC assay. The quantification of apoptosis in MCF-7 and MCF-7/ADR cells.

\section{Figure 7}

TUG1 inhibits Dox resistance by targeting miR-9 in vitro. (A) Apoptosis of MCF-7 cell transfected with miR-9 inhibitor plus a TUG1 siRNA, or miR-9 inhibitor plus a negative control, under different concentrations of Dox according to the PI/Annexin V-FITC assay. (B) The quantification of apoptosis in MCF-7 cells. (C) Proliferation of MCF-7 cells transfected with miR-9 inhibitor plus a TUG1 siRNA, or miR-9 inhibitor plus a negative control oligo, under different concentrations of Dox, according to the EdU assay. (D) The number of EdU-positive cells was counted. (E) Viability of MCF-7 cell transfected with miR-9 inhibitor plus a TUG1 siRNA, or miR-9 inhibitor plus a negative control oligo under different concentrations of Dox, according to the CCK-8 assay. (F) Transfection efficacies of the miR-9 inhibitor in MCF-7 cells. (G) Transfection efficacies of the TUG1 siRNA in MCF-7 cells.

\section{Figure 10}

MiR-9 influences Dox resistance by targeting IncRNA TUG1 in vitro. (A) Transduction of MCF-7 or MCF7/ADR cells with TUG1 siRNA significantly suppressed miR-9 inhibitor-inhibited Dox sensitivity, as determined by CCK-8 assay. (B) Transduction of MCF-7 or MCF-7/ADR cells with TUG1 siRNA significantly suppressed miR-9 inhibitor-promoted proliferation, as determined by EdU assay. (C) The number of EdU-positive cells was counted. (D) Transduction of MCF-7 or MCF-7/ADR cells with TUG1 siRNA significantly suppressed miR-9 inhibitor-inhibited apoptosis, as determined by PI/Annexin V-FITC assay. (E) The quantification of apoptosis in MCF-7 or MCF-7/ADR cells.

\section{Figure 12}

EIF5A2 is the downstream target of IncRNA TUG1. (A) LncRNA TUG1 knockdown down-regulated elF5A2 expression in MCF-7 and MCF-7/ADR cells. (B) Expression of elF5A2 protein in MCF-7 and MCF-7/ADR cells transfected with three TUG1 siRNAs or negative control oligo. (C) Viability of MCF-7 and MCF-7/ADR cells transfected with elF5A2 siRNA plus three TUG1 siRNAs, or elF5A2 siRNA plus a negative control, under different concentrations of Dox, according to the CCK-8 assay. (D) The quantification of apoptosis in MCF-7 cells. (E) Transduction of MCF-7 cells with elF5A2 siRNA significantly suppressed TUG1 siRNApromoted apoptosis, as determined by PI/Annexin V-FITC assay.

\section{Supplementary Files}


This is a list of supplementary files associated with this preprint. Click to download.

- rawimage.tif

- rawimage.tif 